\title{
A semi-parametric approach for imputing mixed data
}

\author{
Irene B. Helenowski* and Hakan Demirtas
}

In this work, we present a semi-parametric method for imputing mixed data which allows us to relax assumptions of the general location model. This approach involves transforming continuous and binary variables to normally distributed data, imputing the data via joint modeling under the normality assumption, and back-transforming the data to their original scale. Transformation and backtransformation of the data comprise the nonparametric portion, and multiple imputation under the normality assumption constitutes the parametric portion of our method. Simulations involving generated mixed data with binary variables and with continuous variables following normal, $t$, Gamma, and mixture Gamma distributions and real data applications indicate promising results, leading us to recommend our approach as a possible avenue for imputing mixed data by semi-parametric means.

\section{INTRODUCTION}

Conventional approaches to imputed mixed data involve joint modeling with components of the normal model for imputing continuous data and of the saturated multinomial or loglinear model for imputing data of the binary or categorical variables (Schafer, 1997) [23]. These approaches, however, rely on assumptions pertaining to the general location model. Here, we propose a new method associated with transforming mixed data consisting of continuous and binary variables to normally distributed data, imputing these data under the normality assumption via joint modeling, and back-transforming the data onto their original scales. These transformations are implemented for continuous variables using principles of the Lurie and Goldberg (1998) [18] algorithm for generation of multivariate continuous data and empirical cumulative distribution function (eCDF) computation. The method given in Barton and Schruben (1993) [1] is involved in back-transformation of eCDF values to the scale of the original data at the final step for imputation of continuous variables. For binary variables, transformation and back-transformations of the data are associated with principles from Emrich and Piedmonte (1991) [14] and Demirtas and Doganay (2012) [9] for binary and mixed data generation, respectively. The backround involving the Lurie

* Corresponding author. and Goldberg (1998) [18] algorithm is discussed in Section 3, eCDF computations are discussed in Section 4, and methods presented in Emrich and Piedmonte (1991) [14] and Demirtas and Doganay (2012) [9] are discussed in Section 5. Our new method is proposed in Section 6 and applications of the method to simulated data and real data examples are included in Sections 7 and 8, respectively. We conclude by recommending our novel approach for imputing mixed data when assumptions regarding the general location model are to be relaxed.

\section{MULTIPLE IMPUTATION}

Multiple imputation, an MCMC (Markov chain Monte Carlo) technique where missing data are replaced with plausible values from a predictive distribution, has increasingly become an attractive option for handling data missing under the MCAR (Missing Completely at Random), MAR (Missing at Random), and MNAR (Missing Not at Random) mechanisms. Under the MCAR mechanism, the probability of missingness does not depend on the missing or observed data, while under the MAR mechanism, the probability may depend on the observed data. Under the MNAR mechanism, missingness depends on the missing data and may or may not depend on the observed data (Rubin, 1987 [22]; Schafer and Olsen, 1998 [25]; Little and Rubin, 2002 [17]; Demirtas and Schafer, 2003 [12]; Demirtas, 2004 [5]; Demirtas, 2004 [6]; Demirtas, 2005 [7]).

Schafer (1997) [23] introduces joint modeling as one approach for imputing data. This technique involved the Expectation-Maximization (EM) and Data Augmentation (DA) algorithms. The EM algorithm is first implemented to provide good starting values for and insight of the convergence behavior of the DA algorithm (Demirtas, 2007 [8]; Demirtas et al., 2008 [13]). The DA algorithm is comprised of two steps: the imputation step, or I-step, given in (1), where values are drawn from a distribution based on the observed data and model parameters, $\theta$, and the posterior step, or P-step, given in (2), where parameters are updated using a distribution based on the observed and imputed data.

$$
\begin{aligned}
Y_{\text {mis }}^{(t+1)} & \sim P\left(Y_{\text {mis }} \mid U_{o b s}, \theta^{(t)}\right) \\
\theta^{(t+1)} & \sim P\left(\theta \mid Y_{o b s}, Y_{\text {mis }}^{(t+1)}\right)
\end{aligned}
$$

where $Y_{o b s}$ are the observed data, $Y_{m i s}$ are the missing data, and are the imputed data. 
Table 1. Simulation results for imputing bivariate mixed data involving a $N(5,1)$ distribution

(Convergence constant used $=0.01$ )

\begin{tabular}{|c|c|c|c|c|c|c|}
\hline \multicolumn{7}{|c|}{ Results for MCAR case } \\
\hline Parameter & True value & $\mathrm{AE}$ & SB & RMSE & $\mathrm{CR}$ & AW \\
\hline $\bar{\delta}$ & -0.7014 & -0.7011 & 14.3375 & 0.0014 & 93.9152 & 0.0901 \\
\hline$\mu_{1}$ & 4.9226 & 4.9410 & & & & \\
\hline$p_{2}$ & 0.4760 & 0.4807 & & & & \\
\hline$\delta$ & -0.3799 & -0.3800 & 3.6626 & 0.0014 & 95.7150 & 0.1513 \\
\hline$\mu_{1}$ & 5.0317 & 5.0259 & & & & \\
\hline$p_{2}$ & 0.5520 & 0.5494 & & & & \\
\hline$\delta$ & 0.4232 & 0.4239 & 41.0072 & 0.0015 & 95.5342 & 0.1451 \\
\hline$\mu_{1}$ & 5.0481 & 5.0498 & & & & \\
\hline$p_{2}$ & 0.5320 & 0.4987 & & & & \\
\hline$\delta$ & 0.7164 & 0.7161 & 14.8059 & 0.0014 & 93.0934 & 0.0864 \\
\hline$\mu_{1}$ & 5.1496 & 5.1531 & & & & \\
\hline$p_{2}$ & 0.4800 & 0.4917 & & & & \\
\hline \multicolumn{7}{|c|}{ Results for MAR case } \\
\hline $\bar{\delta}$ & -0.8197 & -0.8197 & 4.5403 & 0.0004 & 97.3606 & 0.0579 \\
\hline$\mu_{1}$ & 5.0550 & 5.0917 & & & & \\
\hline$p_{2}$ & 0.4920 & 0.5078 & & & & \\
\hline$\delta$ & -0.2215 & -0.2209 & 28.8064 & 0.0016 & 95.7762 & 0.1682 \\
\hline$\mu_{1}$ & 4.9960 & 4.9855 & & & & \\
\hline$p_{2}$ & 0.5360 & 0.5214 & & & & \\
\hline$\delta$ & 0.2881 & 0.2880 & 5.8686 & 0.0014 & 95.9709 & 0.1621 \\
\hline$\mu_{1}$ & 5.0150 & 5.0253 & & & & \\
\hline$p_{2}$ & 0.5040 & 0.4906 & & & & \\
\hline$\delta$ & 0.7388 & 0.7392 & 44.1313 & 0.0008 & 96.7930 & 0.0800 \\
\hline$\mu_{1}$ & 4.9950 & 4.9860 & & & & \\
\hline$p_{2}$ & 0.4440 & 0.4410 & & & & \\
\hline
\end{tabular}

The parameters updated in this algorithm are given in the general location model (Schafer, 1997 [23]; Olsen and Schafer, 2001 [21]). We define this model using $p$ $W_{1}, \ldots, W_{p}$ categorical or binary variables and $q Z_{1}, \ldots, Z_{q}$ continuous variables comprising a data set of dimension $n \times(p+q)$. We then present the two components of the model:

$$
\begin{aligned}
w \mid \pi & \sim M(n, \pi) \\
z_{i} \mid u_{i} & =E_{d}, \mu_{d}, \Sigma \sim N\left(\mu_{d}, \Sigma\right)
\end{aligned}
$$

for $d=1, \ldots, D$, where $\pi$ is the probability that an entry occurs in the $d^{\text {th }}$ cell and $\mu_{d}$ and $\Sigma$ are the mean vector and variance-covariance matrix associated with the distribution of the continuous variables in that $d^{t h}$ cell. Here, $D$ is the number of cells corresponding to possible combination levels among categorical or binary variables, $w$ is a value from a categorical or binary variable following a multinomial distribution, and $z_{i}$ is a value from a continuous variables depending on a mean $\mu_{d}, q \times q$ matrix $\Sigma$ involving $u_{i}=E_{d}$, a $D$ vector of with 1 at position $d$ and 0 elsewhere for $i=$ $1, \ldots, n$.
For example, with three binary variables, there would be $D=2^{3}=8$ cells. Thus, the general location model can be defined in terms of a set of parameters given in (5).

$$
\theta=(\pi, \mu, \Sigma)
$$

The unrestricted model defined above is suitable when the sample size is larger than $D$. When some cells are associated with sparse data or zero counts, however, the means for that cell are omitted from the likelihood, and therefore the maximum likelihood estimate is no longer unique. The problem can be remedied by adding constraints to the model. For example, restrictions can be imposed on categorical variables via the loglinear model:

$$
\log (\pi)=M \lambda
$$

where $\pi$ is the probability vector associated with the categorical variables as before, $M$ is a user-specified matrix, and $\lambda$ is a vector whose first element is used to scale $\pi$ to sum to one. Similarly, we can impose restrictions on continuous variables. To proceed, we first consider the subset of continuous variables in relation to the multivariate regression model: 
Table 2. Simulation results for imputing bivariate mixed data involving a $t_{3}$ distribution (Convergence constant used $=0.01$ )

\begin{tabular}{|c|c|c|c|c|c|c|}
\hline \multicolumn{7}{|c|}{ Results for MCAR case } \\
\hline Parameter & True value & $\mathrm{AE}$ & SB & RMSE & $\mathrm{CR}$ & AW \\
\hline $\bar{\delta}$ & -0.6465 & -0.6470 & 30.1371 & 0.0016 & 93.5712 & 0.1031 \\
\hline$\mu_{1}$ & -0.0827 & -0.0621 & & & & \\
\hline$p_{2}$ & 0.4880 & 0.5060 & & & & \\
\hline$\delta$ & -0.3761 & -0.3756 & 26.0926 & 0.0015 & 95.7219 & 0.1519 \\
\hline$\mu_{1}$ & -0.1206 & -0.11274 & & & & \\
\hline$p_{2}$ & 0.4560 & 0.4553 & & & & \\
\hline$\delta$ & 0.3272 & 0.3273 & 6.6861 & 0.0014 & 96.1203 & 0.1578 \\
\hline$\mu_{1}$ & -0.1285 & -0.1251 & & & & \\
\hline$p_{2}$ & 0.4920 & 0.4898 & & & & \\
\hline$\delta$ & 0.6500 & 0.6504 & 24.0721 & 0.0014 & 94.1362 & 0.1022 \\
\hline$\mu_{1}$ & -0.0446 & -0.0966 & & & & \\
\hline$p_{2}$ & 0.4760 & 0.4539 & & & & \\
\hline \multicolumn{7}{|c|}{ Results for MAR case } \\
\hline $\bar{\delta}$ & -0.6875 & -0.6874 & 6.2143 & 0.0015 & 93.3700 & 0.0936 \\
\hline$\mu_{1}$ & -0.0035 & -0.0582 & & & & \\
\hline$p_{2}$ & 0.5120 & 0.5116 & & & & \\
\hline$\delta$ & -0.2637 & -0.2643 & 32.7540 & 0.0015 & 95.9580 & 0.1643 \\
\hline$\mu_{1}$ & 0.1118 & 0.1516 & & & & \\
\hline$p_{2}$ & 0.5080 & 0.4998 & & & & \\
\hline$\delta$ & 0.2266 & 0.2264 & 12.3985 & 0.0015 & 95.9969 & 0.1676 \\
\hline$\mu_{1}$ & -0.0623 & -0.0886 & & & & \\
\hline$p_{2}$ & 0.4440 & 0.4296 & & & & \\
\hline$\delta$ & 0.7027 & 0.7027 & 0.2119 & 0.0005 & 97.6648 & 0.0892 \\
\hline$\mu_{1}$ & 0.0113 & -0.0751 & & & & \\
\hline$p_{2}$ & 0.5220 & 0.5344 & & & & \\
\hline
\end{tabular}

$$
Z=U \mu+\varepsilon
$$

for $U$ being a $n \times D$ design matrix, $\mu$, a vector of means, $\varepsilon$, the error vector, and $Z$, the matrix of continuous variables, $Z_{1}, \ldots, Z_{q}$, given $W$, the data subset of categorical variables. The mean vector $\mu$ associated with the continuous data can be constrained using:

$$
\mu=A \beta
$$

for some vector $\beta$ and $A$ being a constant matrix of dimension $D \times r$. With the restrictions, the regression model is then defined as:

$$
Z=X \beta+\varepsilon
$$

where $X=U A$ and $\beta$ is associated with a reduced set of regression parameters. The coefficients of the model in equation (9) can therefore be estimable even with zero counts in the contingency table because estimation involves the rank $U A=r<D$, instead of Rank $U=$ $D$, removing cells with zero counts from the computation.

\section{LURIE-GOLDBERG ALGORITHM}

Lurie and Goldberg (1998) [18] developed a method for generating multivariate continuous data using information from marginal distributions and pairwise correlation without requiring information from the joint distribution, which is often unknown. The goal of their algorithm is to minimize $D^{*}$ :

$$
D^{*}=\frac{1}{2} \sum_{i=2}^{k} \sum_{j=1}^{k-1}\left(r_{i j}^{*}-r_{i j}\right)^{2}
$$

with $r_{i j}^{*}$ and $r_{i j}$ as the target correlation matrix and correlation matrix associated with the generated data, respectively. The algorithm involves multiplying a multivariate matrix $\mathbf{X} \sim \mathrm{N}(0, \mathrm{I})$ to the transpose of the lower triangular matrix $\mathbf{L}$ obtained via Cholesky decomposition from the correlation matrix of interest, $\mathbf{R}$, to obtain:

$$
\mathbf{Y}=\mathbf{X} \mathbf{L}^{\mathbf{T}}
$$

Probability distribution function (PDF) values based on the standard normal distribution are then calculated for $\mathbf{Y}$, as given in (12), where $\Phi$ is the cumulative distribution function of the standard normal distribution.

A semi-parametric approach for imputing mixed data 401 
Table 3. Simulation results for imputing bivariate mixed data involving a Gamma $(1,1)$ distribution (Convergence constant used $=0.01$ )

\begin{tabular}{|c|c|c|c|c|c|c|}
\hline \multicolumn{7}{|c|}{ Results for MCAR case } \\
\hline Parameter & True value & $\mathrm{AE}$ & SB & RMSE & $\mathrm{CR}$ & AW \\
\hline $\bar{\delta}$ & -0.6493 & -0.6502 & 46.5629 & 0.0016 & 93.9631 & 0.1023 \\
\hline$\mu_{1}$ & 0.9801 & 0.9848 & & & & \\
\hline$p_{2}$ & 0.5400 & 0.5346 & & & & \\
\hline$\delta$ & -0.3946 & -0.3945 & 8.6858 & 0.0014 & 95.8202 & 0.1493 \\
\hline$\mu_{1}$ & 0.9122 & 0.9021 & & & & \\
\hline$p_{2}$ & 0.4280 & 0.4455 & & & & \\
\hline$\delta$ & 0.4023 & 0.4026 & 17.1405 & 0.0015 & 95.5386 & 0.1482 \\
\hline$\mu_{1}$ & 0.9315 & 0.9286 & & & & \\
\hline$p_{2}$ & 0.5000 & 0.5222 & & & & \\
\hline$\delta$ & 0.6472 & 0.6467 & 27.1875 & 0.0015 & 93.9777 & 0.1031 \\
\hline$\mu_{1}$ & 1.0071 & 0.9672 & & & & \\
\hline$p_{2}$ & 0.5000 & 0.4890 & & & & \\
\hline \multicolumn{7}{|c|}{ Results for MAR case } \\
\hline $\bar{\delta}$ & -0.7125 & -0.7126 & 38.7908 & 0.0002 & 99.1663 & 0.0867 \\
\hline$\mu_{1}$ & 1.1523 & 1.1028 & & & & \\
\hline$p_{2}$ & 0.4500 & 0.4929 & & & & \\
\hline$\delta$ & -0.4550 & -0.4552 & 13.4534 & 0.0015 & 95.2761 & 0.1403 \\
\hline$\mu_{1}$ & 1.0690 & 1.0800 & & & & \\
\hline$p_{2}$ & 0.5040 & 0.5018 & & & & \\
\hline$\delta$ & 0.2568 & 0.2573 & 25.5506 & 0.0015 & 95.9114 & 0.1650 \\
\hline$\mu_{1}$ & 0.9583 & 0.9996 & & & & \\
\hline$p_{2}$ & 0.5300 & 0.5283 & & & & \\
\hline$\delta$ & 0.7103 & 0.7103 & 3.2183 & 0.0013 & 93.5253 & 0.0878 \\
\hline$\mu_{1}$ & 1.0280 & 1.0221 & & & & \\
\hline$p_{2}$ & 0.4720 & 0.4618 & & & & \\
\hline
\end{tabular}

$$
\mathbf{U}=\Phi(\mathbf{Y})
$$

The inverse function of the marginal distribution is next applied to each variable $Y_{j}$, such that:

$$
v_{i j}=F_{j}^{-1}\left(u_{i j}\right)
$$

where the entries $v_{i j}$ comprise the elements of the generated matrix $\mathbf{V}$. The correlation matrix $\mathbf{R}^{*}$, associated with $\mathbf{V}$ can then be computed, i.e.:

$$
\mathbf{R}^{*}=\operatorname{cor}(\mathbf{V})
$$

and compared to the original correlation matrix, $\mathbf{R}$. The algorithm is re-iterated until the quantity defined in (10) is less than some constant $c$ determined by the desired level of accuracy. The criteria in (10) are designed so as to obtain the pairwise associations between variables, along with the marginal distributions desired for each variable.

\section{EMPIRICAL CUMULATIVE DISTRIBUTION FUNCTION (ECDF)}

In addition to principles of the Lurie and Goldberg (1998) [18] algorithm, the portion of our method for imputing con- tinuous data also involved computation of empirical cumulative distribution function (eCDF) values. The purpose of employing eCDF computation is to relax parametric assumptions related to the distribution of the data. We define an eCDF value given in (15) for a real value $x$ as the proportion of values in a random variable less than or equal to $x$, i.e.:

$$
\frac{1}{n} \sum_{i=1}^{n} I\left(X_{i} \leq x\right)
$$

Barton and Schruben (1993) [1] present a method where eCDF values can be mapped to the scale of the original data via:

$$
\begin{aligned}
& F^{-1}\left(u_{C_{(i) j}}\right)=y_{(i) j}+I_{i j}, \\
& I_{i j}=\left(y_{(i+1) j}-y_{(i) j}\right) \frac{u_{C_{(i) j}}-F\left(y_{(i) j}\right)}{F\left(y_{(i+1) j}\right)-F\left(y_{(i) j}\right)}
\end{aligned}
$$

where $F\left(y_{(i) j}\right)$ and $F\left(y_{(i+1) j}\right)$ are the two original eCDF values between which the new eCDF value $u_{C_{(i) j}}$ lies and $y_{(i) j}$ and $y_{(i+1) j}$ are the original data points corresponding to the two original eCDF values. 
Table 4. Imputation results for trivariate data with all variables having missing entries (2 continuous variables, 1 binary variable). Order of Correlations: $\left(Y_{1}, Y_{2}\right),\left(Y_{1}, Y_{3}\right),\left(Y_{2}, Y_{3}\right)$; Order of Means: $Y_{1}, Y_{2}, Y_{3}$

\begin{tabular}{|c|c|c|c|c|c|c|c|c|}
\hline $\begin{array}{l}\text { TRUE } \\
\text { Correlation }\end{array}$ & $\begin{array}{l}\text { Imputed } \\
\text { Correlation }\end{array}$ & SB & RMSE & $\mathrm{CR}$ & AW & $\begin{array}{l}\text { TRUE } \\
\text { Means }\end{array}$ & $\begin{array}{l}\text { Imputed } \\
\text { Means }\end{array}$ & $\begin{array}{l}\text { Convergence } \\
\text { Constant }\end{array}$ \\
\hline \multicolumn{9}{|c|}{$\mathrm{N}(5,1)$ results under MCAR mechanism } \\
\hline-0.7690 & -0.7696 & 14.0590 & 0.0035 & 91.0875 & 0.1653 & 5.0660 & 5.0441 & 0.0250 \\
\hline 0.3473 & 0.3469 & 14.4543 & 0.0021 & 97.3934 & 0.3481 & 5.0540 & 4.9917 & 0.0125 \\
\hline-0.3330 & -0.3312 & 38.2269 & 0.0038 & 95.2266 & 0.3546 & 0.4400 & 0.4519 & 0.0250 \\
\hline \multicolumn{9}{|c|}{$t_{3}$ results under MCAR mechanism } \\
\hline 0.2446 & 0.2463 & 30.3369 & 0.0047 & 94.1530 & 0.3749 & -0.0781 & -0.1031 & 0.0325 \\
\hline-0.5479 & -0.5453 & 47.5926 & 0.0049 & 92.7539 & 0.2827 & 0.2999 & 0.2199 & 0.0325 \\
\hline-0.4037 & -0.4038 & 3.0272 & 0.0047 & 93.3736 & 0.3358 & 0.4900 & 0.4961 & 0.0325 \\
\hline \multicolumn{9}{|c|}{$.75 * \operatorname{Gamma}(5,1)+.25 * \operatorname{Gamma}(1,1)$ results under MCAR mechanism } \\
\hline-0.3350 & -0.3355 & 9.6256 & 0.0038 & 95.1524 & 0.3534 & 0.8804 & 0.8907 & 0.0250 \\
\hline-0.5228 & -0.5216 & 25.4221 & 0.0039 & 94.1745 & 0.2917 & 3.7687 & 3.8261 & 0.0250 \\
\hline 0.4941 & 0.4929 & 29.6774 & 0.0031 & 95.5946 & 0.3021 & 0.5400 & 0.5495 & 0.01975 \\
\hline \multicolumn{9}{|c|}{$\mathrm{N}(5,1)$ results under MCR mechanism } \\
\hline-0.5528 & -0.5531 & 7.6218 & 0.0036 & 94.4052 & 0.2783 & 5.0103 & 5.0141 & 0.0250 \\
\hline 0.4769 & 0.4771 & 5.0039 & 0.0039 & 94.2776 & 0.3091 & 4.9001 & 4.8883 & 0.0250 \\
\hline-0.4936 & -0.4916 & 47.2996 & 0.0038 & 94.8941 & 0.3029 & 0.5700 & 0.5507 & 0.0225 \\
\hline \multicolumn{9}{|c|}{$t_{3}$ results under MAR mechanism } \\
\hline-0.4290 & -0.4298 & 15.3363 & 0.0041 & 94.0607 & 0.3262 & 0.0494 & 0.0080 & 0.0275 \\
\hline 0.3018 & 0.2996 & 42.0112 & 0.0043 & 94.6053 & 0.3628 & 0.4922 & 0.5322 & 0.0275 \\
\hline-0.4294 & -0.4303 & 21.6933 & 0.0034 & 95.2342 & 0.3249 & 0.4000 & 0.4241 & 0.0275 \\
\hline \multicolumn{9}{|c|}{$.75 * \operatorname{Gamma}(5,1)+.25 * \operatorname{Gamma}(1,1)$ results under MAR mechanism } \\
\hline-0.3188 & -0.3205 & 39.8442 & 0.0038 & 95.3493 & 0.3567 & 1.0176 & 0.9910 & 0.0250 \\
\hline 0.4395 & 0.4388 & 19.4776 & 0.0031 & 95.7115 & 0.3216 & 4.1294 & 4.1585 & 0.0225 \\
\hline-0.4988 & -0.5000 & 32.0891 & 0.0032 & 95.4791 & 0.2992 & 0.5600 & 0.5520 & 0.0225 \\
\hline
\end{tabular}

\section{BINARY AND MIXED DATA GENERATION}

Emrich and Piedmonte (1991) [14] and Demirtas and Doganay (2012) [9] discuss multivariate generation of binary and mixed data from multivariate normally distributed values, respectively. In introducing these methods, we first present some pairwise correlation coefficients, such as the phi, tetrachoric, and point-biserial correlations. The phi correlation, given as:

$$
\frac{n_{11} n_{00}-n_{10} n_{01}}{\sqrt{\left(n_{10}+n_{11}\right)\left(n_{00}+n_{01}\right)+\left(n_{00}+n_{10}\right)\left(n_{01}+n_{11}\right)}}
$$

for $n_{j k}=\mathrm{I}\left(Y_{1}=j, Y_{2}=k\right)$, is a special case of the Pearson correlation measuring the association between two binary variables. The phi correlation is related to the tetrachoric correlation via:

$$
\Phi\left[z\left(p_{j}\right), z\left(p_{k}\right), \rho_{j k}\right]=\delta_{j k}\left(p_{j} q_{j} p_{k} q_{k}\right)^{1 / 2}+p_{j} p_{k}
$$

where the tetrachoric correlation measures the association of two normally distributed variables underlying the binary variables. Here, $\rho_{j k}$ is the tetrachoric correlation, $\delta_{j k}$ is phi correlation coefficient, $\Phi$ is the bivariate normal CDF with mean 0 , standard deviation 1 , and correlation $\rho_{j k}, p_{j}$ and $p_{k}$ are the proportion parameters for $Y_{j}$ and $Y_{k}$, respectively, $q_{j}=1-p_{j}$ and $q_{k}=1-p_{k}$. Lastly, $z\left(p_{j}\right)$ and $z\left(p_{k}\right)$ are the quantile functions of the standard normal distribution for $p_{j}$ and $p_{k}$, respectively.

Emrich and Piedmonte (1991) [14] indicate that the multivariate normally distributed data generated using a correlation matrix with the pairwise tetrachoric correlations can then be dichotomized by quantiles based on proportions corresponding to the desired binary data. The third correlation we discuss here, the point-biserial correlation, assesses the relationship between a binary variable and a continuous variable. It is related to the Pearson correlation measuring the association between two continuous variables by:

$$
\begin{aligned}
& \delta_{Y_{1} Y_{2 D}}=\left(\frac{h}{\sqrt{p(1-p)}}\right) \rho_{Y_{1} Y_{2}}, \\
& p=\operatorname{Pr}\left(Y_{2 D}=1\right)
\end{aligned}
$$

where $Y_{2}$ is a normally distributed variable underlying the binary variable $Y_{2 D}$ and $h$ is the ordinate of the normal curve at the point defined for the binary split of $Y_{2 D}$ (Demirtas and Doganay, 2012) [9].

A semi-parametric approach for imputing mixed data 403 
Table 5. Imputation results for trivariate data with all variables having missing entries (1 continuous variable, 2 binary variables). Order of Correlations: $\left(Y_{1}, Y_{2}\right),\left(Y_{1}, Y_{3}\right),\left(Y_{2}, Y_{3}\right)$; Order of Means: $Y_{1}, Y_{2}, Y_{3}$

\begin{tabular}{|c|c|c|c|c|c|c|c|c|}
\hline $\begin{array}{l}\text { TRUE } \\
\text { Correlation }\end{array}$ & $\begin{array}{l}\text { Imputed } \\
\text { Correlation }\end{array}$ & SB & RMSE & $\mathrm{CR}$ & AW & $\begin{array}{l}\text { TRUE } \\
\text { Means }\end{array}$ & $\begin{array}{l}\text { Imputed } \\
\text { Means }\end{array}$ & $\begin{array}{l}\text { Convergence } \\
\text { Constant }\end{array}$ \\
\hline \multicolumn{9}{|c|}{$\mathrm{N}(5,1)$ results under MCAR mechanism } \\
\hline-0.3968 & -0.3948 & 36.2180 & 0.0044 & 94.0132 & 0.3377 & 5.0230 & 5.0494 & 0.0250 \\
\hline-0.3999 & -0.4001 & 4.9991 & 0.0040 & 94.3233 & 0.3357 & 0.5300 & 0.5287 & 0.0250 \\
\hline 0.2859 & 0.2869 & 18.7734 & 0.0042 & 94.3767 & 0.3663 & 0.4400 & 0.4340 & 0.0250 \\
\hline \multicolumn{9}{|c|}{$t_{3}$ results under MCAR mechanism } \\
\hline-0.2179 & -0.2155 & 48.1378 & 0.0043 & 94.9570 & 0.3795 & -0.0724 & -0.0257 & 0.02500 \\
\hline-0.3667 & -0.3657 & 18.6737 & 0.0043 & 94.2560 & 0.3460 & 0.4800 & 0.4682 & 0.02675 \\
\hline 0.3126 & 0.3121 & 9.6684 & 0.0043 & 94.3174 & 0.3603 & 0.5400 & 0.5590 & 0.02675 \\
\hline \multicolumn{9}{|c|}{$\operatorname{Gamma}(5,1)$ results under MCAR mechanism } \\
\hline-0.2991 & -0.2975 & 27.0884 & 0.0047 & 93.5797 & 0.3648 & 5.4220 & 5.4124 & 0.0275 \\
\hline-0.3703 & -0.3676 & 48.3907 & 0.0048 & 93.9201 & 0.3459 & 0.5200 & 0.5288 & 0.0275 \\
\hline 0.2358 & 0.2382 & 42.6390 & 0.0049 & 93.8312 & 0.3769 & 0.5100 & 0.5098 & 0.0275 \\
\hline \multicolumn{9}{|c|}{$\mathrm{N}(5,1)$ results under MAR mechanism } \\
\hline-0.2811 & -0.2804 & 13.5379 & 0.0040 & 94.9069 & 0.3668 & 5.1270 & 5.0899 & 0.0275 \\
\hline 0.4799 & 0.4803 & 8.7371 & 0.0038 & 94.2611 & 0.3082 & 0.4900 & 0.4927 & 0.0275 \\
\hline-0.1908 & -0.1904 & 9.4503 & 0.0034 & 95.9627 & 0.3820 & 0.5400 & 0.5210 & 0.0275 \\
\hline \multicolumn{9}{|c|}{$t_{3}$ results under MAR mechanism } \\
\hline-0.3066 & -0.3081 & 35.3747 & 0.0036 & 95.6176 & 0.3596 & -0.1576 & -0.1073 & 0.02325 \\
\hline 0.5322 & 0.5303 & 45.9748 & 0.0035 & 94.9354 & 0.2876 & 0.4500 & 0.4967 & 0.02325 \\
\hline-0.2891 & -0.2899 & 19.1129 & 0.0035 & 95.6721 & 0.3638 & 0.4400 & 0.4325 & 0.02325 \\
\hline \multicolumn{9}{|c|}{$\operatorname{Gamma}(5,1)$ results under MAR mechanism } \\
\hline-0.3180 & -0.3162 & 40.0162 & 0.0041 & 95.0151 & 0.3582 & 4.7573 & 4.7396 & 0.0275 \\
\hline 0.2696 & 0.2706 & 21.8347 & 0.0038 & 95.3798 & 0.3684 & 0.5100 & 0.5223 & 0.0275 \\
\hline-0.2932 & -0.2908 & 47.5888 & 0.0046 & 94.5414 & 0.3647 & 0.3400 & 0.3838 & 0.0275 \\
\hline
\end{tabular}

Demirtas and Doganay (2012) [9] extend the method of Emrich and Piedmonte (1991) [14] to generate multivate mixed data. They use principles from Emrich and Piedmonte (1991) [14] to generate binary variables. Namely, they first generate a multivariate normally distributed data set using a correlation matrix with tetrachoric pairwise correlations as defined in (18) corresponding to pairs of two binary variables, point-biserial pairwise correlations, as given in (19), corresponding to pairs of a binary and a normally distributed variable, and pairwise correlations corresponding to pairs with two normally distributed variables. After generating the multivariate normally distributed data, Demirtas and Doganay (2012) [9] then dichotomize variables designated as binary and rescale variables designated as normally distributed variables using equation (20).

$$
Z_{l}=a_{l} Y_{l}+b_{l}, \quad l=1, \ldots, q
$$

where $a_{l}$ and $b_{l}$ correspond to the scale and location parameters of the desired distribution of variable $Z_{l}$.

\section{NEW METHOD}

We introduce our method by discussing the transformation of continuous variables and binary variables to normally distributed variables, separately. With the continuous variables, we first compute the eCDF values for the observed data and obtain corresponding normally distributed values using the inverse function of the standard normal distribution:

$$
\mathrm{Y}^{*}=\Phi^{-1}(\mathrm{U})
$$

With the binary variables, we first compute the pairwise phi coefficients as given in (17) and apply equation (18) to these coefficients in order to obtain the pairwise tetrachoric correlations. We next determine if the correlation matrix is positive semi-definite. If the matrix is not positive semi-definite, we can find the positive semi-definite matrix "closest" to this matrix (Higham, 2002) [16]. We use the matrix with pairwise tetrachoric correlations to generate multivariate normally distributed data and introduce the same fraction of missing information in the multivariate normally distributed data as is present in the original binary data.

Multiple imputation via joint modeling under the normality assumption is then applied to a data set with these normally distributed values corresponding to the continuous variables and the binary variables combined. The 
Table 6. Imputation results for 4-variable data with $Y_{2}$ having missing entries (2 continuous variables, 2 binary variables)

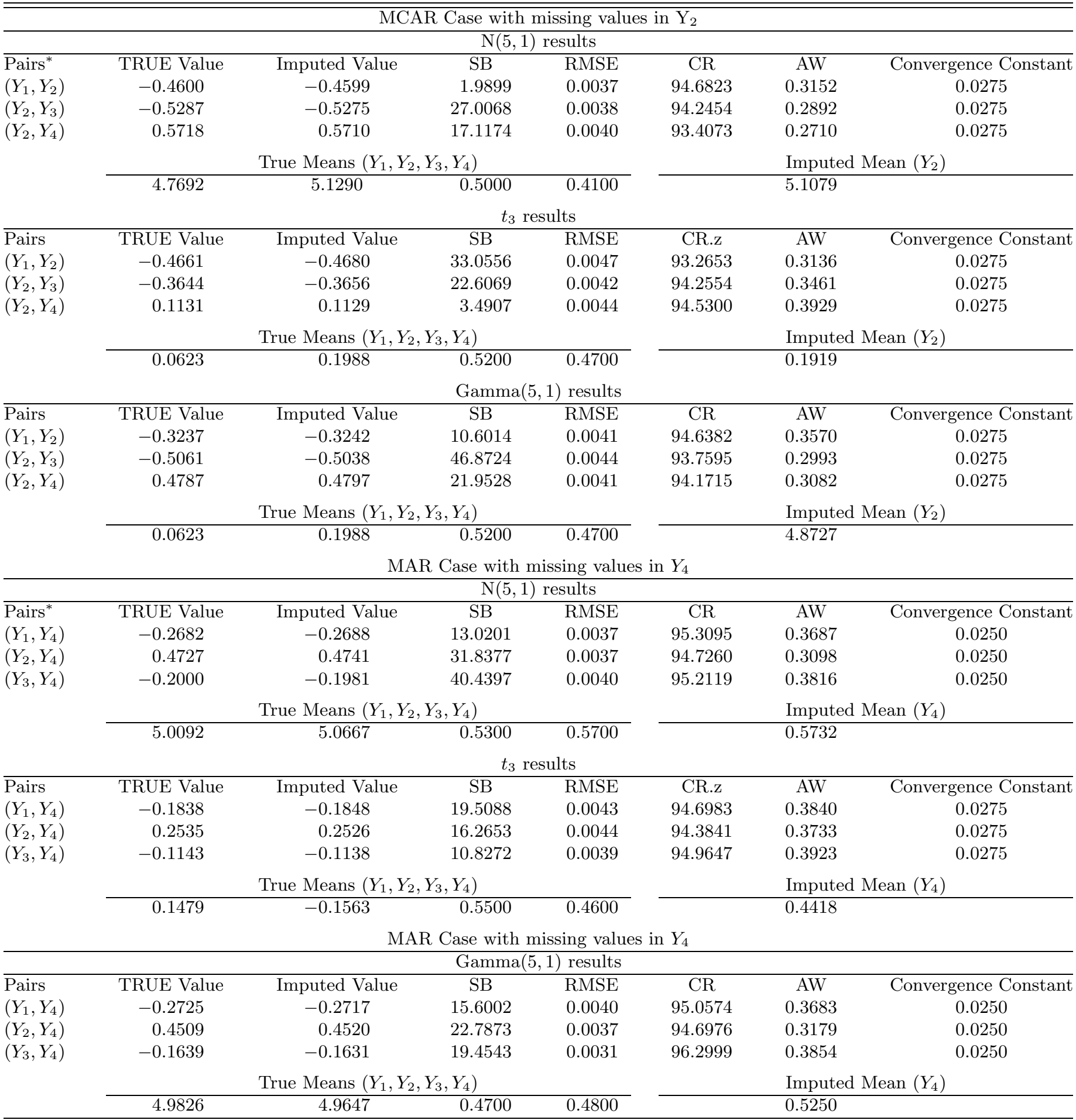

marginal PDF values of the imputed data based on the for each variable $Y_{j}$ following the distribution marginal distribution of the imputed data are then obtained, $N\left(\mu_{j}^{* i m p}, \sigma_{j}^{* i m p}\right)$.

i.e.:

$$
Y_{j}^{* i m p} \sim N\left(\mu_{j}^{* i m p}, \sigma_{j}^{* i m p}\right)
$$

We next apply the equation given in (16) in order to map the imputed values pertaining to the continuous variable onto the scale of the original data. The imputed values 
Table 6. (Continued)

\begin{tabular}{|c|c|c|c|c|c|c|c|}
\hline \multicolumn{8}{|c|}{ MAR Case with missing values in $Y_{2}$} \\
\hline \multicolumn{8}{|c|}{$\mathrm{N}(5,1)$ results } \\
\hline Pairs* & TRUE Value & Imputed Value & SB & RMSE & $\mathrm{CR}$ & AW & Convergence Constant \\
\hline$\left(Y_{1}, Y_{2}\right)$ & -0.2588 & -0.2595 & 14.2597 & 0.0040 & 95.0299 & 0.3711 & 0.0275 \\
\hline$\left(Y_{2}, Y_{3}\right)$ & 0.2444 & 0.2431 & 26.7741 & 0.0039 & 95.1759 & 0.3741 & 0.0275 \\
\hline$\left(Y_{2}, Y_{4}\right)$ & -0.2393 & -0.2402 & 17.8786 & 0.0038 & 95.1718 & 0.3749 & 0.0275 \\
\hline \multicolumn{5}{|c|}{ True Means $\left(Y_{1}, Y_{2}, Y_{3}, Y_{4}\right)$} & \multicolumn{3}{|c|}{ Imputed Mean $\left(Y_{4}\right)$} \\
\hline & 5.0051 & 5.0110 & 0.4400 & 0.4700 & & 4.9711 & \\
\hline \multicolumn{8}{|c|}{$t_{3}$ results } \\
\hline Pairs & TRUE Value & Imputed Value & SB & RMSE & CR.z & AW & Convergence Constant \\
\hline$\left(Y_{1}, Y_{2}\right)$ & -0.3283 & -0.3296 & 31.7107 & 0.0035 & 95.6598 & 0.3543 & 0.0250 \\
\hline$\left(Y_{2}, Y_{3}\right)$ & 0.2936 & 0.2944 & 18.3900 & 0.0035 & 95.5753 & 0.3630 & 0.0250 \\
\hline$\left(Y_{2}, Y_{4}\right)$ & -0.2501 & -0.2482 & 45.3306 & 0.0036 & 95.8699 & 0.3723 & 0.0250 \\
\hline \multicolumn{5}{|c|}{ True Means $\left(Y_{1}, Y_{2}, Y_{3}, Y_{4}\right)$} & \multicolumn{3}{|c|}{ Imputed Mean $\left(Y_{2}\right)$} \\
\hline & 0.0666 & -0.5757 & 0.5500 & 0.5300 & & -0.5750 & \\
\hline \multicolumn{8}{|c|}{$\operatorname{Gamma}(5,1)$ results } \\
\hline Pairs & TRUE Value & Imputed Value & SB & RMSE & $\mathrm{CR}$ & AW & Convergence Constant \\
\hline$\left(Y_{1}, Y_{4}\right)$ & -0.3062 & -0.3054 & 17.3540 & 0.0035 & 95.5676 & 0.3604 & 0.0258 \\
\hline$\left(Y_{2}, Y_{4}\right)$ & 0.4598 & 0.4590 & 16.8437 & 0.0038 & 94.6741 & 0.3154 & 0.0258 \\
\hline$\left(Y_{3}, Y_{4}\right)$ & -0.3820 & -0.3838 & 38.9096 & 0.0040 & 94.9327 & 0.3399 & 0.0258 \\
\hline & \multicolumn{4}{|c|}{ True Means $\left(Y_{1}, Y_{2}, Y_{3}, Y_{4}\right)$} & \multicolumn{3}{|c|}{ Imputed Mean $\left(Y_{4}\right)$} \\
\hline & 4.7025 & 4.9320 & 0.4800 & 0.5300 & & 4.8578 & \\
\hline
\end{tabular}

pertaining to binary variables are lastly dichotomized by quantiles corresponding to probabilities computed for the binary data by:

$$
\begin{aligned}
& \operatorname{Pr}\left(Y_{k}=y_{k} \mid Y_{1}=y_{1}, Y_{2}=y_{2}, \ldots, Y_{k-1}=y_{k-1}, R\right), \\
& R=\left\{R_{1}, R_{2}, \ldots, R_{K-1}, R_{K}\right), \\
& y_{k}=0,1 ; \quad k=1, \ldots, K
\end{aligned}
$$

where $R_{1}, \ldots, R_{k}$ are the missingness indicators for $Y_{1}, \ldots, Y_{k}$. The probabilities defined in (23) are computed by the equation below.

$$
\begin{aligned}
& \sum I\left(Y_{k}=1 \mid Y_{1}, Y_{2}, \ldots, Y_{k-1}, R_{1}, R_{2}, \ldots, R_{k}\right) \\
& =\sum \frac{I\left(Y_{k}=1, Y_{1}, Y_{2}, \ldots, Y_{k-1}, R_{1}, R_{2}, \ldots, R_{k}\right)}{I\left(Y_{1}, Y_{2}, \ldots, Y_{k-1}, R_{1}, R_{2}, \ldots, R_{k}\right)}
\end{aligned}
$$

The reason behind this computation is to determine the probability of the binary value for the missing entry within a subset of the data set given what other binary variables are observed.

The imputed continuous variables and the imputed binary variables are then again combined. Next, the pairwise correlation between imputed continuous variables, the pairwise phi correlations between imputed binary variables and the point-biserial correlations between imputed continuous and binary variables via equation (19) are calculated and compared to the respective biserial, phi, and point-biserial pairwise correlations ob- tained from the original data via the following equation:

$$
\left|\delta_{j k}-\delta_{j k}^{i m p}\right|<c_{j k}
$$

where $\delta_{j k}^{i m p}$ and $\delta_{j k}$ are the pairwise correlations obtained from the imputed and original data, respectively and $c_{j k}$ is some constant chosen to achieve standardized bias values $<50 \%$ and coverage rates $>90 \%$ for each pairwise correlation between variables $Y_{j}$ and $Y_{k}$. The algorithm is reiterated until (25) is satisfied for all pairwise correlations. Note that the selection of $c_{i j}$ is data-specific, as it depends on the incomplete data under consideration.

We summarize the steps of our algorithm with the following diagram (Figure 1), where we note the steps at which that the continuous and binary variables are separated before transformation to normally distributed values, recombined for multiple imputation, separated for backtransformation onto the original scales, and then recombined again to create the final data set.

\section{SIMULATION STUDY}

We examine our method for imputing mixed data using several different bivariate and multivariate examples. Simulation studies involved generating data under MCAR and MAR mechanism with pairwise correlations ranging from -0.75 to 0.75 and including continuous variables which followed the normal, $t$, Gamma, or mixed Gamma distributions. Such settings were chosen to examine the applica- 
Table 7. Characteristics and imputation results including assessment measures for pairwise correlations involving Prostate SPORE bivariate mixed data. Biopsy cores positive for Cancer is the continuous with 49 missing values is involved in each case. Convergence constant used $=0.01$

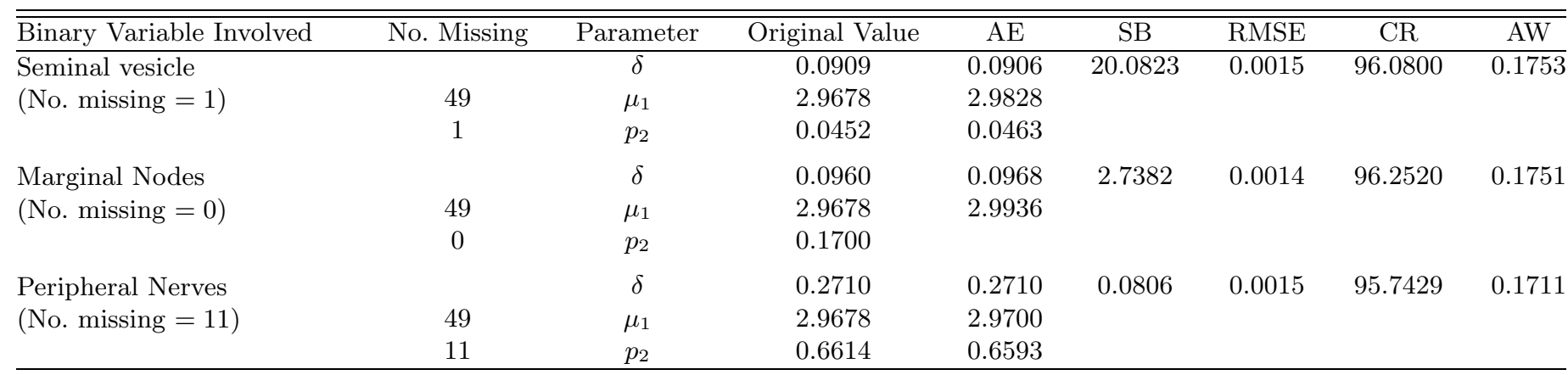

Table 8. Variables in 100 men in the Prostate SPORE database used in the imputation application to multivariate mixed data and estimates and average estimates and assessment measures from the imputation application to multivariate mixed data from the Prostate SPORE database

\begin{tabular}{|c|c|c|c|c|c|c|}
\hline Variable & \multicolumn{4}{|c|}{ Label } & \multicolumn{2}{|c|}{ Number (Percent) Missing } \\
\hline$\overline{Y_{1}}$ & \multicolumn{4}{|c|}{ Percent Cancer in Prostate Gland } & \multicolumn{2}{|c|}{$0(10.0 \%)$} \\
\hline$Y_{2}$ & \multirow{2}{*}{\multicolumn{4}{|c|}{$\begin{array}{c}\text { Percent Biopsy Cores Positive for Prostate Cancer } \\
\text { Marginal nodes (positive vs. negative) }\end{array}$}} & \multicolumn{2}{|c|}{$19(19.0 \%)$} \\
\hline$Y_{3}$ & & & & & & \\
\hline Pairs & Original correlation & Imputed correlation & SB & RMSE & $\mathrm{CR}$ & AW \\
\hline$\overline{\left(Y_{1}, Y_{2}\right)}$ & 0.3366 & 0.3362 & 8.6253 & 0.0035 & 95.3717 & 0.353 \\
\hline$\left(Y_{2}, Y_{3}\right)$ & 0.264 & 0.2651 & 22.7556 & 0.004 & 95.0477 & 0.3699 \\
\hline
\end{tabular}

$Y_{\text {cont }}$

1. $\rightarrow U_{\text {con }}$

2. $\rightarrow Y_{\text {cont }}^{*}$

$Y_{\text {bin }}$

3. $\rightarrow Y_{b i n}^{*} \quad Y_{b i n}^{* i m p}$

4. $Y^{*}=\left(Y_{\text {cont }}^{*}, Y_{\text {bin }}^{*}\right)$

5. $\rightarrow Y^{* i m p}$

$Y_{\text {cont }}^{* i m p}$
6. $\rightarrow U_{c o n t}^{i m p}$
7. $\rightarrow Y_{\text {cont }}^{i m p}$
$Y_{\text {bin }}^{* i m p}$
8. $\rightarrow Y_{\text {bin }}^{i m p}$
9. $Y^{i m p}=\left(Y_{\text {cont }}^{i m p}, Y_{\text {bin }}^{i m p}\right)$

Figure 1. Diagram indicating the steps for the new procedure of imputing mixed data, including how continuous and binary variables are separated, transformed to normally distributed values, combined, imputed, separated again, back-transformed onto the scale of the original data and finally recombined.

tion of our method to data missing under either mechanism and involving pairwise correlations of different magnitudes. Furthermore, distributions associated with continuous variables were chosen to show that our method can be applied to data with continuous variables following any distribution, whether symmetric (normal), symmetric but heavy-tailed $(t)$, or skewed (Gamma). Bivariate data sets include 500 entries and multivariate data sets include 100 entries. Here, the number of entries refers to the number of observations and the number of missing values in each variable. Thus, for example, in bivari- ate cases, there are two variables and each variable contains a number of observations and a number of missing values which sum to 500. Each bivariate data set included one binary variable and one continuous variable. In both bivariate and multivariate cases, binary and continuous variables were generated separately; these variables were then combined into one data set and pairwise correlations were introduced. In multivariate examples, we generated data sets with three variables (having two continuous variables and one binary variable or one continuous variable and two binary variables) and four variables (with two continuous and two binary variables). All continuous variables involved in one multivariate data set follow the same distribution.

Under the MCAR mechanism, 25\% of entries were deleted in the bivariate, trivariate, and 4 -variable cases. To generate missing data in the second variable in bivariate cases under the MAR mechanism, missingness was induced in the binary variable dependent on the continuous variable via equation (26), where $R_{Y_{2}}=0$ indicates that $Y_{2}$ is missing.

$$
\begin{aligned}
& \log \left(\frac{p_{2}}{1-p_{2}}\right)=-2.75+0.25 Y_{1}, \\
& p_{2}=\operatorname{Pr}\left(R_{Y_{2}}=0\right)
\end{aligned}
$$

Missing values in the trivariate and 4 -variable data sets were introduced in the second variable under the MAR mechanism using equation (27). 

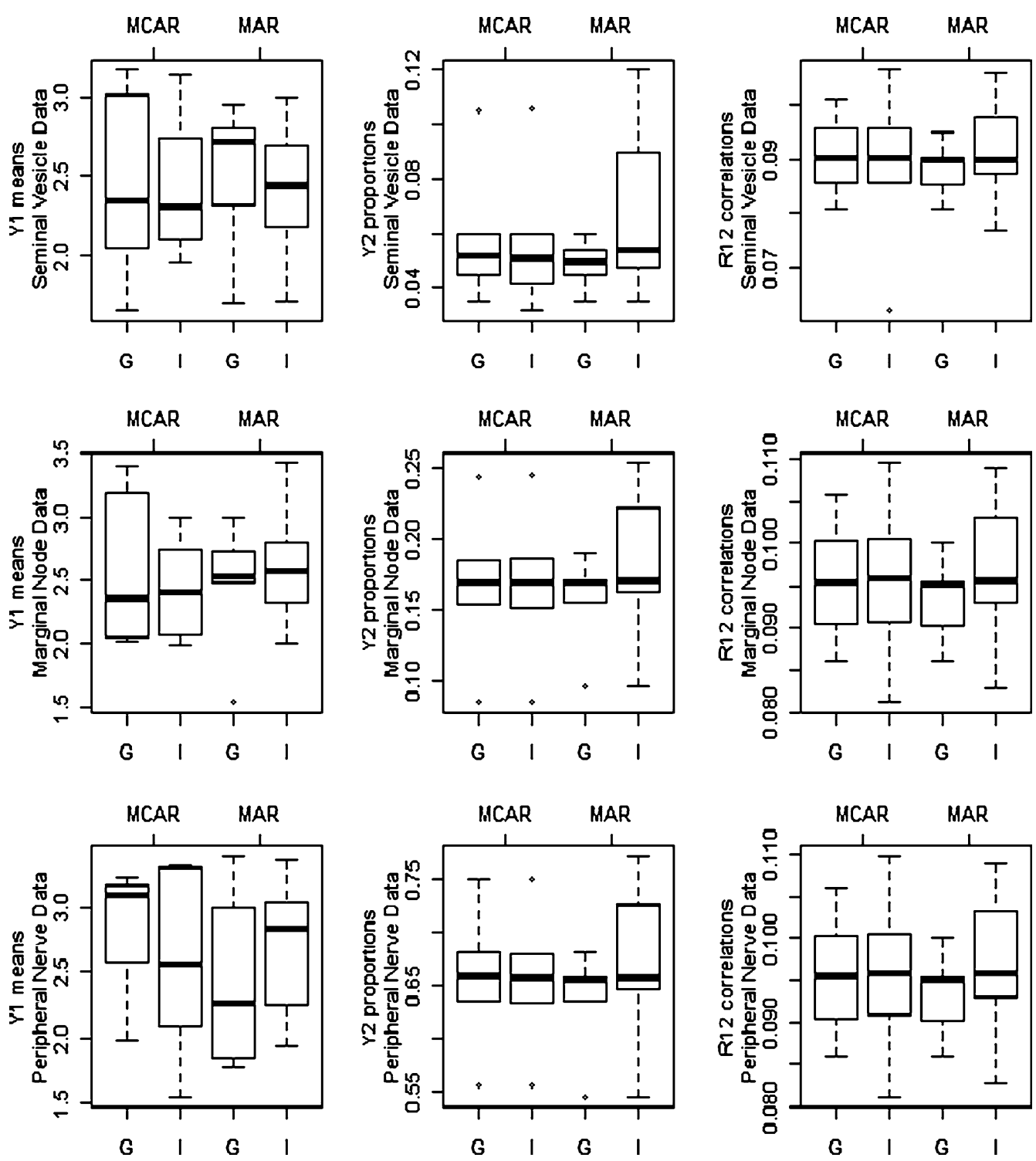

Figure 2. Boxplots of $Y_{1}$ means, $Y_{2}$ proportions, and pairwise correlations between $Y_{1}$ and $Y_{2}$ obtained from generated $(G)$ and imputed (I) bivariate data sets resembling real data involving variables for number of biopsy cores positive for cancer $\left(Y_{1}\right)$ and presence or absence of cancer in seminal vesicles, marginal nodes, or peripheral nerves $\left(Y_{2}\right)$. Missing values were introduced under the MCAR mechanism in both variables or under the MAR mechanism in the binary variable and under the MCAR mechanism in the continuous variable.

$$
\begin{aligned}
& \log \left(\frac{p_{2}}{1-p_{2}}\right)=-0.5+0.00025 Y_{3}, \\
& p_{2}=\operatorname{Pr}\left(R_{Y_{2}}=0\right)
\end{aligned}
$$

such that the probability of missingness depended on the third variable, $Y_{3}$. Missing values were then introduced in $Y_{1}$ and $Y_{3}$ under the MCAR mechanism by randomly deleting $25 \%$ of the entries in each of those variables.

We assessed the performance of our method via assessment measures for the pairwise correlations which included average estimate $(\mathrm{AE})$, standardized bias (SB), the root mean square error (RMSE), the coverage rate $(\mathrm{CR})$, and average width (AW) of the confidence intervals. The average estimate (AE) is the average of all parameter estimates obtained from all imputed data sets across all simulations. Standardized bias (SB), an accuracy measure, given in (28), is defined as the absolute difference between the true parameter and parameter estimate obtained from the simulations divided by the standard deviation obtained from the simulations; satisfactory SB values should be less than $50 \%$. A standardized bias value of $50 \%$ indicates that the discrepancy between the estimate and true value is $1 / 2$ the magnitude of the standard deviation, corresponding to $1 / 8$ th of a typical confidence interval which is considered accept- 

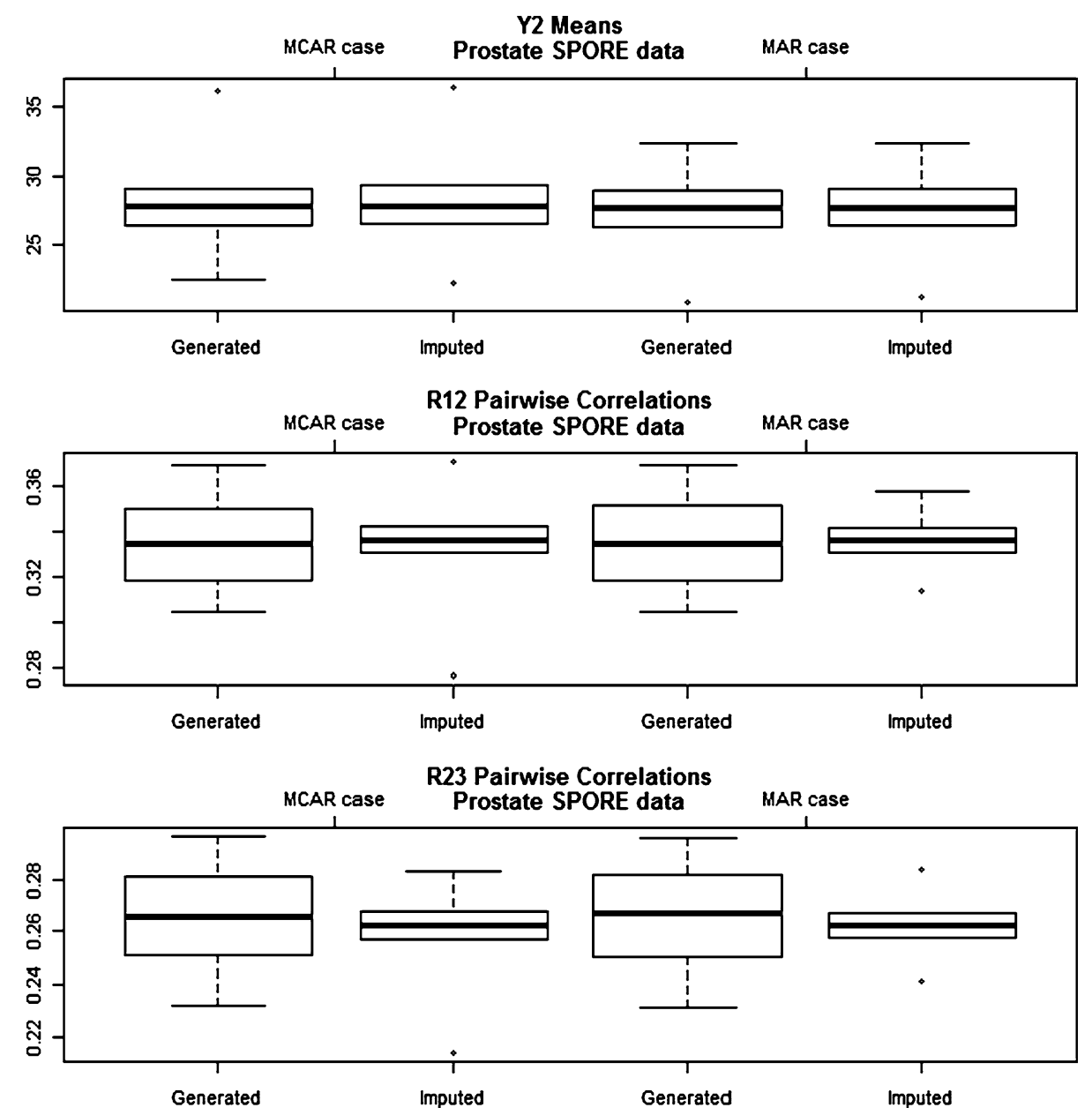

Figure 3. Boxplots of means and pairwise correlations involving percent biopsy cores positive for prostate cancer $\left(Y_{2}\right)$ obtained from generated and imputed data sets for the multivariate real data examples where missing data were introduced in $Y_{2}$ via the MCAR or MAR mechanisms.

able. The root mean square error (RMSE), given in (29), is used to evaluate precision and accuracy with small values being preferable. The coverage rate $(\mathrm{CR})$, again used to evaluate precision and accuracy, is defined as the percentage of times that the confidence interval of parameter estimate obtained from imputed data encompasses the true parameter, where rates $>90 \%$ indicate sufficient coverage. Lastly, average width (AW), a precision measure, involves the average difference between the lower and upper bound of the confidence limits for the parameter estimate across all imputations (Collins et al., 2001 [4]; Schafer and Graham, 2002 [24]; Demirtas and Hedeker, 2007 [19]; Demirtas and Hedeker, 2008 [11]; Demirtas et al., 2008 [13]).

$$
\begin{aligned}
& 100 \times\left|\frac{E(\theta-\widehat{\theta})}{S E(\widehat{\theta})}\right| \\
& \sqrt{E_{\theta}(\widehat{\theta}-\theta)^{2}}
\end{aligned}
$$

Results indicate that our method performs adequately in both bivariate (Tables 1, 2, and 3) and multivariate cases (Tables 4,5 , and 6 ) as shown by AE values comparable to true parameters, SB estimates $<50 \%$, small RMSE values, CR values $>90 \%$, and AW values comparable to the $95 \%$ confidence interval widths of the true parameters for pairwise correlations.

\section{REAL DATA EXAMPLE}

Our real data example includes variables coming from the Prostate SPORE database. The Specialized Program of Research Excellence in Prostate Cancer (SPORE Grant \#: P50 Ca 090386), established in 2001, is a collaborative effort between Northwestern University, the University of Chicago, and Northshore University Health System facilities aimed to develop new approaches for prostate cancer prevention, diagnosis, and treatment. The database contains demographic, clinical, and pathological information from 3,452 

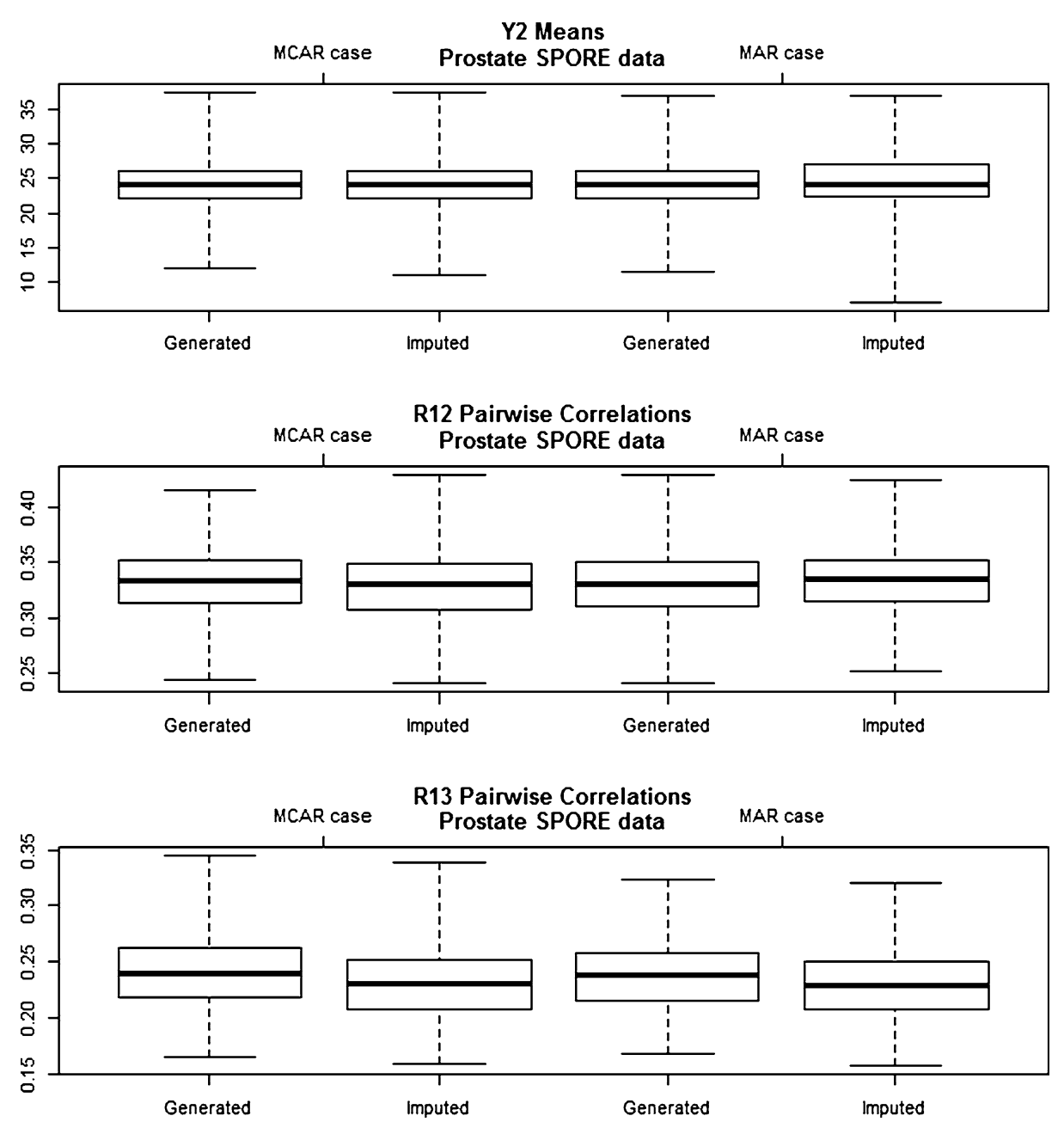

Figure 4. Boxplots of means and pairwise correlations involving percent biopsy cores positive for prostate cancer $\left(Y_{2}\right)$ obtained from generated and imputed data sets for the multivariate real data examples where missing data were introduced in $Y_{2}$ via the MCAR or MAR mechanisms. Ranges for pairwise correlations from generated data and imputed data are comparable when the convergence criteria pertaining to those correlations are relaxed.

men as of 2011. Original estimates are obtained from the incomplete data. Previous analyses of these data were conducted to determine confounding factors which could contribute to bias and none were found.

Tables 7 and 8 give the descriptions of data sets used in our bivariate and multivariate examples. Here, the number of biopsies staining positive for cancer, the percentage of biopsies staining positive for cancer, and the percentage of cancer in the removed prostate gland are continuous variables, and presence of cancer in seminal vesicles, marginal nodes, or peripheral nerves are binary variables. Furthermore, the first two variables mentioned above involve data collected from needle biopsies, while the other variables were obtained from radical prostatectomy. The association between data from biopsies and from radical prostatectomy is of interest to investigators with respect to prostate cancer diagnosis as biopsy is a preferable alternative to radical prostatectomy (Mazzuchelli et al., 2005 [19]; Montironi et al., 2008 [20]; Bill-Axelson et al., 2011 [2]). With biopsy data, both number and percent positive staining have important biological implications in prostate cancer research (Gancarczyk et al., 2003 [15]; Bittner et al., 2010 [3]). Therefore, investigators often wish to examine the relationship between prognostic factors and both these variables.

Results in Tables 7 and 8 again indicate promise in the application of our method via $\mathrm{AE}$ values comparable to original estimates, SB estimates $<50 \%$, small RMSE values, CR estimates $>90 \%$, and AW values comparable to the $95 \%$ confidence interval widths of original estimates for the pairwise correlations.

We further generated 1,000 data sets with characteristics similar to the real data examples. The same fraction of missing information was introduced in these generated data sets as found in the original data. This missingess was in- 
troduced under the MCAR mechanism via random deletion or under the MAR mechanism using equations:

$$
\begin{aligned}
& \log \left(\frac{p_{1}}{1-p_{1}}\right)=-1.125+0.0005 Y_{2}, \\
& p_{1}=\operatorname{Pr}\left(R_{Y_{1}}=0\right)
\end{aligned}
$$

in the bivariate cases and:

$$
\begin{aligned}
& \log \left(\frac{p_{2}}{1-p_{2}}\right)=-2.0-0.0005 Y_{3}, \\
& p_{2}=\operatorname{Pr}\left(R_{Y_{2}}=0\right)
\end{aligned}
$$

in the multivariate cases where one can see that missingness in a continuous variable depended on a binary variable in each case. We applied our method to each data set involving 10 imputations. Boxplots in Figures 2 and 3 indicate that means and proportions from generated and imputed data sets are acceptably comparable. Pairwise correlations for imputed data are associated with slightly narrower ranges as our multiple imputation algorithm is designed to converge when the pairwise correlations from the imputed data are sufficiently close to those of original pairwise correlations. When the convergence criteria dependent on the pairwise correlations are relaxed, i.e., when a larger $c_{i j}$ was chosen, the ranges of the estimates from the generated and from the imputed data appear more comparable (Figure 4).

\section{CONCLUSION}

In this work, we present a semi-parametric approach for imputing mixed data which adopts principles from the Lurie and Goldberg (1998) [18] algorithm for generating multivariate continuous data, eCDF computation, and the Demirtas and Doganay (2012) [9] algorithm for generating mixed data, which in turn includes principles of the Emrich and Piedmonte (1991) [14] algorithm for generating binary data. Namely, principles found in Lurie and Goldberg (1998) [18] and of eCDF computation were involved in the imputation of continuous variables while imputation of binary variables was associated with principles of generating binary and mixed data as discussed in Emrich and Piedmonte (1991) [14] and Demirtas and Doganay (2012) [9]. In the latter case, pairwise phi correlations measuring the associations of binary variables were used to derive pairwise tetrachoric correlations involved in generating the multivariate normally distributed data to be imputed. eCDF computation was incorporated into our algorithm, to relax parametric assumptions on the distribution of the data. eCDF, by construction, is invariant to changes in scale and location.

This imputation technique differs from the random number generation methods in that the imputed normally distributed variables designated as continuous are mapped onto the scale of the original data via nonparametric means as found in the approach given in Barton and Schruben (1993) [1]. Simulation studies and real data applications of our method led to promising results. Therefore, we propose this approach as a possible avenue for multiple imputation of mixed data when assumptions of the general location model need to be relaxed.

\section{ACKNOWLEDGMENTS}

The authors would like to thank Drs. Donald Hedeker, Sally Freels, Hua Yun Chen, and Borko Jovanovic for their input and recommendations in developing this approach and preparing this work. This work has been supported by the Northwestern University SPORE in Prostate Cancer (Grant \#: P50 Ca 090386).

Received 8 October 2012

\section{REFERENCES}

[1] Barton, R. R. and Schruben, L. W. (1993). Uniform and Bootstrap Resampling of Empirical Distributions. In Proceedings of the 25th Conference on Winter Simulation (1993), pp. 503-508.

[2] Bill-Axelson, A., Holmberg, L., Ruutu, M., Garmo, H., Stark, J. R., Busch, C., Nordling, S., Haggman, M., Andersson, S-O., Bratell, S., Spangberg, A., Palmgren, J., SteiNeCK, G., Adami, H-O., and Johansson, J-E. (2011). Radical Prostatectomy Versus Watchful Waiting in Early Prostate Cancer. New Engl. J. Med. 364 1708-1717.

[3] Bittner, N., Merric, G. S., Galbreath, R. W., Bulter, W. M., Adamovich, E., and Wallner, K. E. (2010). Greater Biopsy Core Number Is Associated with Improved Biochemical Control in Patients Treated with Permanent Prostate Brachytherapy. Int. J. Radiation Oncology Biol. Phys. 78 1104-1110.

[4] Collins, L. M., Schafer, J. L., and Kam, C-M. (2001). A Comparison of Inclusive and Restrictive Strategies in Modern Missing Data Procedures. Psychological Methods 6 330-351.

[5] Demirtas, H. (2004). Modeling Incomplete Longitudinal Data. Journal of Modern Applied Statistical Methods 3 305-321.

[6] Demirtas, H. (2004). Simulation Driven Inferences for Multiply Imputed Longitudinal Data Sets. Statistica Neerlandica 58 446482. MR2113212

[7] Demirtas, H. (2005). Multiple Imputation Under Bayesianly Smoothed Pattern-Mixture Models for Non-ignorable Drop-Out. Statistics in Medicine 24 2345-2363. MR2151710

[8] Demirtas, H. (2007). Practical Advice on How to Impute Continuous Data when the Ultimate Interest Centers on Dichotomized Outcomes Through Pre-Specified Thresholds. Communications in Statistics - Simulation \& Computation 36 871-889. MR2415691

[9] Demirtas, H. and Doganay, B. (2012). Simultaneous Generation of Binary and Normal Data with Specified Marginal and Association Structures. Journal of Biopharmaceutical Statistics 22 223-236. MR2880620

[10] Demirtas, H. and Hedeker, D. (2007). Gaussianization-Based Quasi-Imputation and Expansion Strategies for Incomplete Correlated Binary Responses. Statistics in Medicine 26 782-799. MR2339174

[11] Demirtas, H. and Hedeker, D. (2008). Multiple Imputation Under Power Polynomials. Communications in Statistics - Simulation $\&$ Computation 37 1682-1695. MR2542425

[12] Demirtas, H. and Schafer, J. L. (2003). On the Performance of Random-Coefficient Pattern-Mixture Models for Non-ignorable Drop-Out. Statistics in Medicine 22 2553-2575. 
[13] Demirtas, H. Freels, S. A., and Yucel, R. M. (2008). Plausibility of Multivariate Normality Assumption when Imputing Non-Gaussian Continuous Ouctomes: A Simulation Assessment. Journal of Statistical Computation and Simulation 78 69-84. MR2420089

[14] Emrich, J. L. and Piedmonte, M. R. (1991). A Method for Generating High-Dimensional Multivariate Binary Variates. The American Statistician 45 302-304.

[15] Gancarczyk, K. J., Wu, H., Mcleod, D. G., Kane, C., Kusuda, L., Lance, R., Herring, J., Foley, J., Baldwin, D., Bishoff, J. T., Soderalh, D., and Moul, J. W. (2003). Using the Percentage of Biopsy Cores Positive for Cancer, Pretreatment PSA, and Highest Biopsy Gleason Sum to Predict Pathologic Stage after Radical Prostectomy: The Center for Prostate Disease Research Nonograms. Urology 61 588-595.

[16] Higman, D. G. (1987). Coherent Algebras. Linear Algebra Appl. 93 209-239. MR0898557

[17] Little, R. J. A. and Rubin, D. B. (2002). Statistical Analysis with Missing Data, 2nd ed. Wiley, New York. MR1925014

[18] Lurie, P. M. and Goldberg, M. S. (1998). An Approximate Method for Sampling Correlated Random Variables from Partially-Specified Distributions. Management Science 44203 218.

[19] Mazzucchelli, R., Barbisan, F., Tarquini, L. M., Filosa, A., Campanini, N., and Galosi, A. B. (2005). Gleason Grading of Prostate Carcinoma in Needle Biopsies vs. Radical Prostatectomy Specimens. Anal. Quant. Cytol. Histol. 27 125-133.

[20] Montironi, R., Cheng, L., and Beltran, A. L. (2008). Editorial Comment on: Comparing the Gleason Prostate Biopsy and Gleason Prostatectomy Grading System: The Lahey Clinic Medical Center Experience and an International Meta-Analysis. European Urology 54 371-381.
[21] Olsen, M. K. and Schafer, J. L. (2001). A Two-Part RandomEffects Model for Semicontinuous Longitudinal Data. Journal of the American Statistical Association 96 730-745. MR1946438

[22] Rubin, D. B. (1987). Multiple Imputation for Nonresponse in Surveys. John Wiley, New York. MR0899519

[23] Schafer, J. L. (1997). Analysis of Incomplete Multivariate Data. Chapman and Hall, London. MR1692799

[24] Schafer, J. L. and Graham J. W. (2002). Missing Data: Our View of the State of the Art. Psychological Methods 7 147-177.

[25] Schafer, J. L. and Olsen M. K. (1998). Multiple Imputation for Multivariate Missing Data Problems: A Data Analyst's Perspective. Multivariate Behavioral Research 33 545-571.

Irene B. Helenowski

Feinberg School of Medicine

Northwestern University

Department of Preventive Medicine

680 N. Lake Shore Drive, Suite 1400

Chicago, IL 60611-4407

USA

E-mail address: i-helenowski@northwestern.edu

\section{Hakan Demirtas}

University of Illinois at Chicago

School of Public Health (MC 923)

1603 W. Taylor Street

Chicago, IL 60612-4336

USA

E-mail address: demirtas@uic.edu 